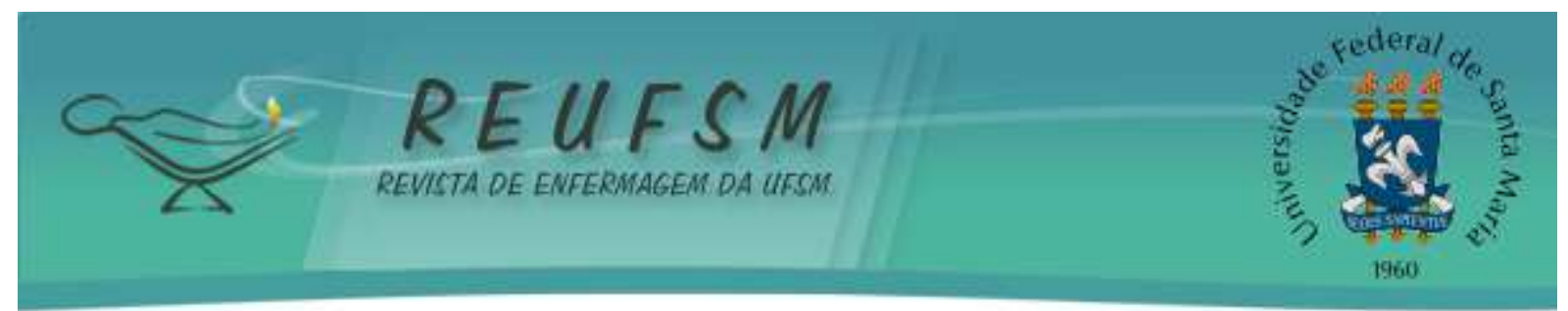

\title{
SABERES E PRÁTICAS DOS PROFISSIONAIS DE SAÚDE NA ATENÇÃO À PESSOA COM DIABETES MELLITUS
}

\section{KNOWLEDGE AND PRACTICE OF HEALTH PROFESSIONALS IN THE CARE OF PEOPLE WITH DIABETES MELLITUS}

\section{CONOCIMIENTO Y PRÁCTICAS DE PROFESIONALES DE SALUD EN LA ATENCIÓN A LA PERSONA CON DIABETES MELLITUS}

\author{
Priscila Juceli Romanoski ${ }^{1}$ \\ Denise Maria Guerreiro Vieira da Silva ${ }^{2}$ \\ Julia Estela Willrich Böell ${ }^{3}$ \\ Maria Elena Echevarria Guanilo ${ }^{4}$ \\ Francieli Lohn da Rocha ${ }^{5}$ \\ Thais Silva Pereira Campos ${ }^{6}$
}

Doi: $10.5902 / 2179769230931$

RESUMO: Objetivo: compreender os saberes e as práticas dos profissionais de saúde na atenção primária em relação ao atendimento à pessoa com Diabetes Mellitus (DM) e suas complicações. Método: exploratório descritivo qualitativo, conduzido em cinco centros de saúde do município de Florianópolis, Santa Catarina. Realizada entrevista semiestruturada com 22 profissionais de saúde vinculados à Estratégia Saúde da Família e ao Núcleo de Apoio à Saúde da Família, entre dezembro de 2014 a maio de 2015. Os resultados foram analisados na perspectiva de análise de conteúdo convencional. Resultados: o saber dos profissionais configura-se no atendimento integral e humanizado, com foco na qualidade de vida. Suas práticas assistenciais se distanciam desses saberes quando a pessoa já apresenta a doença. Conclusões: apesar de os participantes manifestarem saberes convergentes com as políticas públicas, a prática clínica desenvolvida na assistência à pessoa com DM exige reflexão e estruturação, a fim de retardar ou evitar complicações.

Descritores: Diabetes mellitus; Atenção primária à saúde; Profissionais de saúde; Doença crônica; Políticas públicas

ABSTRACT: Aim: to understand the knowledge and practices of health professionals in primary care for patients with diabetes mellitus and its complications. Method: descriptive qualitative,

\footnotetext{
1 Enfermeira, Mestre em Enfermagem, Doutoranda do Programa de Pós-Graduação em Enfermagem da Universidade Federal de Santa Catarina. Florianópolis, Santa Catarina, Brasil. Email: priscila.romanoski@gmail.com

${ }^{2}$ Enfermeira, Doutora em Enfermagem, Professora Associado do Departamento de Enfermagem e do Programa de Pós Graduação em Enfermagem da Universidade Federal de Santa Catarina. Florianópolis, Santa Catarina, Brasil. E-mail: denise_guerreiro@hotmail.com

${ }^{3}$ Educadora física, Enfermeira, Doutora em Enfermagem pelo Programa de Pós-Graduação em Enfermagem da Universidade Federal de Santa Catarina. Florianópolis, Santa Catarina, Brasil. E-mail: juliaestela_8@hotmail.com

${ }^{4}$ Enfermeira, Doutora em Enfermagem, Professora do Departamento de Enfermagem e do Programa de Pós Graduação em Enfermagem da Universidade Federal de Santa Catarina. Florianópolis, Santa Catarina, Brasil. Email: elena_meeg@hotmail.com

${ }^{5}$ Enfermeira, Imperial Hospital de Caridade de Florianópolis. Florianópolis, Santa Catarina, Brasil. E-mail: franh_lr@hotmail.com

${ }^{6}$ Enfermeira, Mestre em Enfermagem, Docente no Programa de Graduação em Enfermagem da Faculdade Guanambi. Vitória da Conquista, Bahia, Brasil. E-mail: taisinhasilva@gmail.com
} 


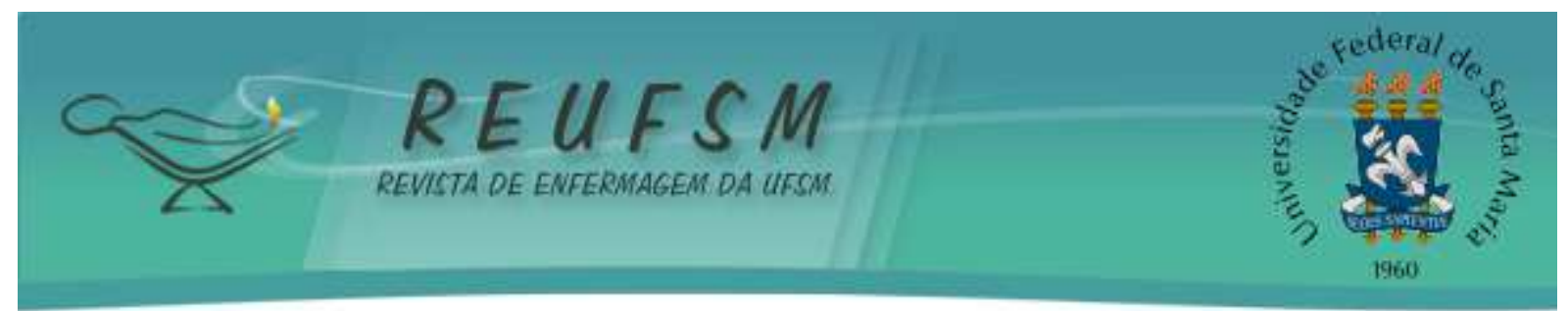

exploratory study conducted in five health centers in the city of Florianópolis, Santa Catarina. A semi-structured interview was conducted with 22 health professionals linked to the Family Health Strategy and to the Family Health Support Center between December 2014 and May 2015. The results were analyzed from the perspective of conventional content analysis. Results: the knowledge of professionals is formed through an integrated and humanized care, focusing on quality of life. Their care practices are distanced from such knowledge when the person already has the disease. Conclusions: although the participants manifest knowledge that converge with public policies, the clinical practice developed in the assistance of people with DM requires reflection and structuring in order to delay or avoid complications.

Descriptors: Diabetes mellitus; Primary Health Care; Health Personnel; Chronic Disease; Public Policy

RESUMEN: Objetivo: comprender el conocimiento y las prácticas de los profesionales de salud en la atención primaria a las personas con diabetes mellitus y sus complicaciones. Método: exploratorio, descriptivo, cualitativo; desarrollado en cinco Centros de Salud, de la ciudad de Florianópolis, Santa Catarina. Se realizó entrevista semiestructurada con 22 profesionales de la Estrategia de Salud de la Familia y del Núcleo de Apoyo de la Salud de la Familia, entre diciembre/2014 y mayo/2015. Los resultados fueron analizados a partir de la perspectiva de análisis de contenido convencional. Resultados: el saber de los profesionales se configura por la atención integral y humanizada, con énfasis en la calidad de vida. Sus prácticas asistenciales se distancian de esos saberes cuando la persona ya presenta la enfermedad. Conclusiones: aunque los participantes tengan conocimientos convergentes a los de las políticas públicas, la práctica clínica requiere reflexión y estructuración para retrasar o evitar sus complicaciones.

Descriptores: Diabetes mellitus; Atención primaria de salud; Profesionales de la salud; Enfermedad crónica; Políticas públicas

\section{INTRODUÇÃO}

As Doenças Crônicas Não Transmissíveis (DCNT), em muitos países, são o principal problema de saúde pública e representam a maior causa de morte, acometendo homens e mulheres, das mais variadas idades. No Brasil, atingem $72,4 \%$ das pessoas adultas. ${ }^{1}$ E, dentre as DCNT, destaca-se o Diabetes Mellitus (DM), visto as estatísticas indicaram, a cada dez segundos, duas pessoas são diagnosticadas com DM e uma morre de causa relacionada a essa. Atualmente, há mais de 415 milhões de pessoas com DM no mundo; mas, estima-se que esse número aumente para 642 milhões até $2040 .^{2}$

Em 2010 havia 10 milhões de pessoas com DM no Brasil. A estimativa é que, no ano de 2025, sejam 17,6 milhões de indivíduos com essa. ${ }^{3}$ Segundo a Federação Internacional de Diabetes, aproximadamente 12 milhões de brasileiros, entre 20 e 79 anos, convivem com o DM, isso coloca o país na quinta posição em um ranking de causa mundial de mortes. ${ }^{4-6}$ 


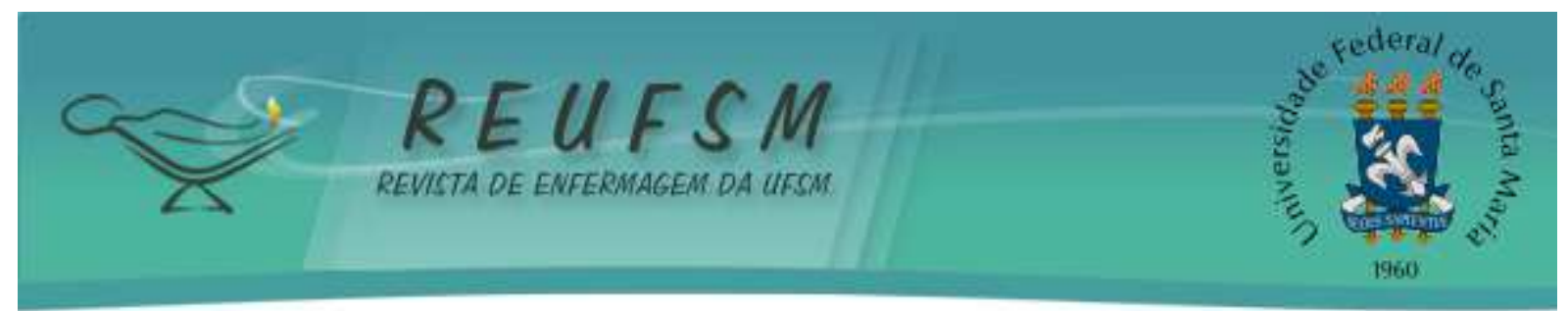

As pessoas, ao conviverem com uma condição crônica de saúde, necessitam de cuidados contínuos, os quais implicam custos elevados e resultam em sofrimentos que, muitas vezes, poderiam ser retardados ou evitados se houvesse atenção apropriada dos profissionais de saúde. Uma dessas situações que pode ser prevenida ou ter seu aparecimento retardado refere-se às complicações crônicas do DM; tanto as macrovasculares quanto as microvasculares, incluindo as neuropáticas. ${ }^{5,7-8}$

Frente à complexidade dos elementos implicados no cuidado integral do paciente com DM, o profissional de saúde precisa estar preparado para atuar no sentido de oferecer as melhores opções de controle da condição crônica e evitar as possíveis complicações. O acompanhamento sistemático, o acolhimento e a formação do vínculo; a disponibilização de medicamentos e outros insumos necessários, de acordo com as necessidades e especificidades; a educação em saúde, individual e coletiva utilizando abordagens adequadas, são instrumentos indispensáveis para os profissionais atuarem com as pessoas com DM. ${ }^{9}$

Com o foco nas doenças crônicas, o Ministério da Saúde (MS) tem proposto o desenvolvimento de programas, protocolos e materiais educativos para auxiliar o profissional de saúde na correta implantação do modelo assistencial da Atenção Básica (AB). Exemplos importantes são os Cadernos de Atenção Básica: Estratégias para o cuidado da pessoa com doença crônica e Estratégias para o cuidado da pessoa com doença crônica - Diabetes Mellitus, disponíveis online e/ou impressos, que são instrumentos que valorizam as práticas de saúde na atenção à pessoa com DM. ${ }^{9-10}$

Outra ação do MS foi a criação das Redes de Atenção à Saúde (RAS), para integrar e organizar as ações e os serviços de saúde. ${ }^{11}$ Em 2013, o MS propôs diretrizes para o cuidado das pessoas com doenças crônicas nas redes de atenção à saúde e nas linhas de cuidado prioritárias. ${ }^{10}$ A Portaria $N^{\circ} 483$, de $1^{\circ}$ de abril de 2014, redefiniu a RAS das Pessoas com Doenças Crônicas visando melhorar a atenção à saúde desses indivíduos, sobretudo aquele com DM. ${ }^{12}$ Objetivou-se alavancar a mudança do modelo de atenção à saúde, por meio do fortalecimento da atenção às pessoas com doenças crônicas, garantindo o cuidado integral, impactando positivamente nos indicadores relacionados a essas doenças, promovendo a saúde da população e prevenindo o desenvolvimento desses agravos e suas complicações. ${ }^{13}$

Essas medidas objetivam a promoção da saúde, a prevenção e o retardo das complicações do DM e desenvolvem programas específicos com cuidados centrados na atenção ao cuidado da 


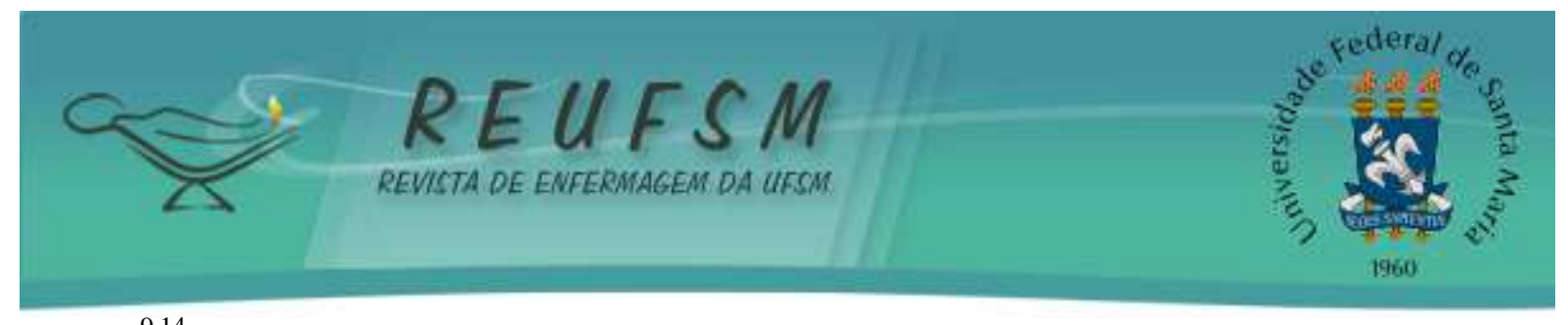

doença. ${ }^{9,14}$ Nessa perspectiva, questionou-se: "quais são os saberes e as práticas dos profissionais de saúde que atuam na Atenção Primária à Saúde sobre atenção às pessoas com DM e suas complicações?" Assim, observa-se compreender os saberes e as práticas dos profissionais de saúde na atenção primária em relação ao atendimento à pessoa com DM e suas complicações.

\section{MÉTODO}

Trata-se de um estudo exploratório descritivo de abordagem qualitativa a partir dos dados da dissertação de mestrado intitulada: Saberes e práticas dos profissionais de saúde sobre a atenção às pessoas com diabetes mellitus, ${ }^{15}$ apoiado pela agência de fomento Coordenação de Aperfeiçoamento de Pessoal de Nível Superior (CAPES).

Participaram do estudo 22 profissionais de saúde pertencentes a cinco centros de saúde e do município de Florianópolis, Santa Catarina. Foi selecionado um centro de cada distrito sanitário, conforme a organização da Rede de Atenção Primária deste município. Os profissionais eram vinculados à Estratégia Saúde da Família (ESF) e ao Núcleo de Apoio à Saúde da Família (NASF) que atendiam pessoas com DM. A coleta de dados ocorreu de dezembro de 2014 a maio de 2015 e a técnica utilizada foi a entrevista semiestruturada.

No primeiro contato, foi apresentada a pesquisa ao coordenador do centro de saúde, que indicou os profissionais que seriam os possíveis participantes, considerando aqueles que atendiam aos seguintes critérios de inclusão: (i) ter, no mínimo, um ano de experiência em atenção primária, (ii) ter vínculo empregatício e atuação no centro de saúde, (iii) ser profissional com graduação na área da saúde. O único critério de exclusão foi estar em afastamento ou férias no período da coleta de dados. A interrupção da coleta de dados se deu a partir do momento em que os relatos não trouxeram novas informações relevantes para a pesquisa.

Os profissionais foram contatados e, posteriormente ao aceite, foi agendada a entrevista no centro de saúde, ficando a critério do profissional o dia e horário. Mediante leitura, explicação e assinatura do Termo de Consentimento Livre e Esclarecido em duas vias, foi iniciada a entrevista, com a busca de vínculo com a pesquisadora. Foi enfatizado interesse em ouvir o participante e esclarecido que seriam utilizadas perguntas norteadoras, sendo a questão inicial: "fale sobre sua atuação no seu local de trabalho em relação às pessoas com DM?”. A duração média foi de 40 minutos, todas foram gravadas em áudio digital, a partir da anuência dos participantes. 


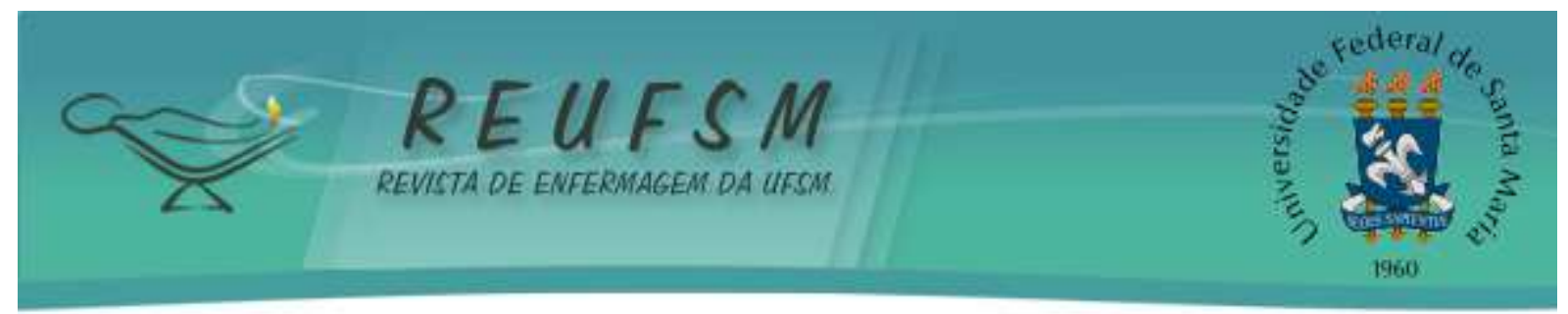

As entrevistas foram transcritas, em editor de texto, pela pesquisadora principal e por bolsistas de iniciação científica. Para auxiliar na organização dos dados, foi utilizado o software Etnhograph 6.0. Os dados foram analisados na perspectiva de análise de conteúdo convencional proposta por Hsieh \& Shannon, a qual orienta os pesquisadores a evitar o uso de categorias préconcebidas, permitindo que categorias e subcategorias surjam a partir dos dados. ${ }^{16}$

Inicialmente, foi realizada uma leitura atenta de todos os dados repetidamente para atingir a imersão e noção do todo. Em seguida, as informações das 22 entrevistas foram codificadas linha a linha, sendo identificados, no primeiro processo, 565 códigos.

Os códigos foram organizados e reunidos por semelhança, resultando em 25 subcategorias, as quais foram sintetizadas em cinco categorias. Os resultados foram organizados em dois temas: "saberes dos profissionais de saúde sobre o atendimento à pessoa com DM" e "práticas dos profissionais de saúde desenvolvidas nos centros de saúde".

Para preservar o anonimato dos participantes, empregou-se o sistema alfanumérico para identificação dos seus relatos (P01 a P22), realizando-se também identificações por profissões, sendo enfermeiro (ENF); médico (MED); dentista (DEN); educador físico (EDF); nutricionista (NUT) e farmacêutico (FAR) e pelos centros de saúde (C1 a C5).

Durante todo o processo de pesquisa, foram respeitados os aspectos éticos da Resolução n ${ }^{\circ}$ 466/12 do Conselho Nacional de Saúde do Ministério da Saúde ${ }^{17}$ e foi obtida a autorização do Comitê de Ética em Pesquisa com Seres Humanos da Universidade Federal de Santa Catarina, em 08 de julho de 2014, sob o parecer $n^{\circ} 711.982$.

\section{RESULTADOS}

Participaram do estudo dez enfermeiros, cinco médicos, dois educadores físicos, dois nutricionistas, dois dentistas e um farmacêutico. Quanto ao tempo de experiência em atuação na atenção básica, prevaleceu o de um a cinco anos - 14 participantes; de seis a dez anos cinco; mais de dez anos de experiência - três. Outra característica importante foi a formação complementar, sendo que 16 pessoas relataram ter Especialização em Saúde da Família; três em Residência em Saúde da Família; dois em Doenças Crônicas Não Transmissíveis; dois relataram ter desenvolvido mestrado em Saúde Coletiva/Pública. E cinco possuíam outras formações não relacionadas diretamente à temática. 


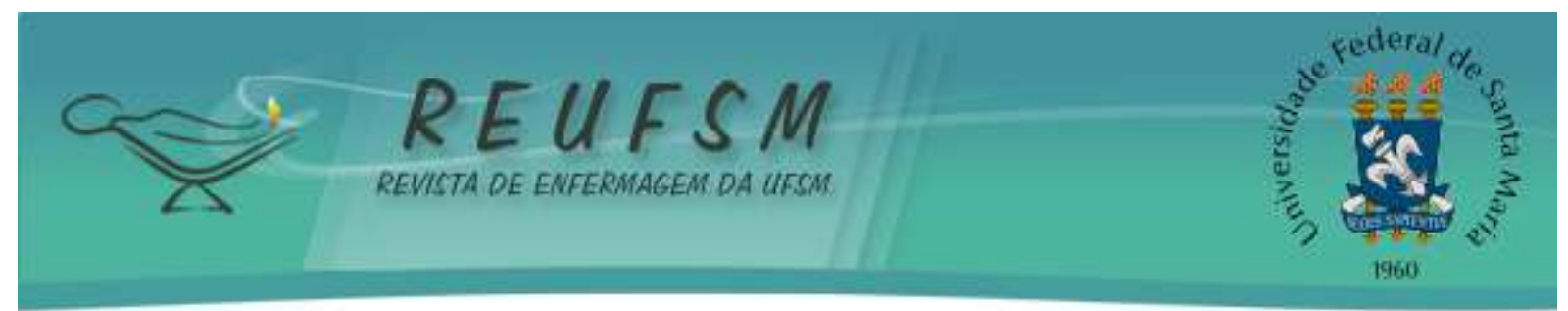

Os saberes dos profissionais apontaram a relevância do atendimento integral à pessoa com DM, porque o contexto em que convive e sua condição global de saúde precisam ser considerados. Os profissionais de saúde demonstraram valorizar a abordagem interdisciplinar e os múltiplos aspectos envolvidos na assistência em saúde e que promovem a qualidade de vida da pessoa com DM. O atendimento humanizado foi relatado por meio de vínculo, respeito, empatia e preocupação em não gerar sentimento de culpa e, sim, em manter a esperança da pessoa com DM.

Os profissionais enfatizaram a importância do compromisso com a segurança do paciente, de compartilhar as decisões com outros profissionais para complementar esse conjunto de ações, conforme ilustra o seguinte relato.

Às vezes, o paciente descompensado precisa medicar na hora. Chama a médica. E ela atende aqui junto comigo e já resolve, resolve na hora, ou chama a nutricionista, ou encaminha para ela dar um suporte. (P13 ENF C3)

Os profissionais mostraram ter sensibilidade e preocupação com os sentimentos gerados a partir de suas orientações na atenção à pessoa com DM. A culpa ou medo que a pessoa com DM trazia consigo era um desses que preocupava os profissionais na adesão ao tratamento. Reconheciam que decorriam, muitas vezes, era causado por suas orientações ou pela descoberta do diagnóstico.

Acho que a gente pode lidar de uma forma indireta, ou direta, [...] que amedronte menos. Porque todo mundo tem um estigma no diabético. Quando você diz que a pessoa é diabética, é duro de ouvir, né? [...] Às vezes, um fardo. (P07 MED C1)

Verifica-se que eles entendiam que era importante manter a esperança das pessoas e não as culpar ou amedrontar com a possibilidade de terem complicações. Esse entendimento podia estar relacionado à formação complementar em Saúde da Família, visto que mais da metade dos entrevistados possuía especialização nessa área.

Foi possível constatar que os profissionais buscaram formação em atenção primária visando melhorar o atendimento e utilizavam os documentos do MS como referência para a condução dos cuidados e os tratamentos que realizavam. As trocas de experiências entre os profissionais também foram valorizadas como forma de obter mais conhecimentos e, possivelmente, influenciaram nas suas concepções sobre a atenção à pessoa com DM.

Alguns profissionais relataram que muitas vezes acabavam não lendo ou retomando os documentos do MS que serviriam para orientar sua prática. Esses profissionais tinham 


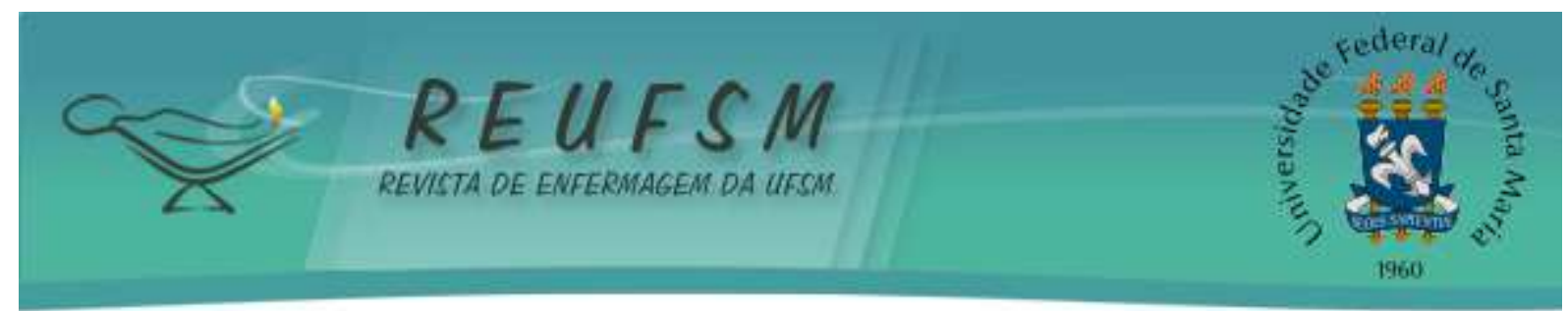

consciência de que as informações trazidas pela mídia exerciam influência nas pessoas, sendo uma ferramenta que ainda precisava ser explorada para auxiliar na busca de novos casos e na corresponsabilidade dos indivíduos nos cuidados com sua saúde.

Esses cadernos de atenção básica me ajudavam bastante. Ultimamente eu estou bem paradinha com leituras, não leio mais nada. Mais a internet, é a primeira fonte de pesquisa [...] (P16 FAR C4)

Eu não fui mais atrás de capacitação [...] É assim, se eu não me deparar com essas situações, a gente não procura. Então, no momento eu acho que eu não fui mais atrás de material para me informar sobre diabetes. (P19 ODO C3)

Quando a informação chega, a demanda, a procura é maior. O ruim é essa informação chegar de forma inadequada. (P09 ENF C2)

Os profissionais ressaltaram a importância de focar na qualidade de vida da pessoa e não na doença. Isso mostrou que a atenção ao cuidado vem mudando na concepção de um atendimento mais voltado indivíduo.

Acho que a primeira coisa, em trabalhar com o doente crônico, é você não trabalhar o foco na doença, e sim na qualidade de vida da pessoa. (P05 MED C1)

No momento eu não trabalho com grupo específico para diabéticos, mas grupo de caminhada [...] que iniciou em razão do grupo de diabéticos [...] e surgiu a necessidade de fazer um grupo de atividade física para eles e hoje é aberto para outras pessoas também (P06 EDF C1)

As práticas dos profissionais de saúde ainda não são desenvolvidas de acordo com a concepção do seu saber, compreendendo as necessidades trazidas pela condição crônica. Segundo eles, a estrutura do sistema de saúde os distanciava do que consideravam a atenção ideal.

Havia um esforço em atender a grande demanda no cotidiano, o que dificultava a efetividade do controle, o acompanhamento e a busca ativa das pessoas com DM. Enfatizaram que o acolhimento era realizado com maior frequência pelo enfermeiro e que esse profissional tinha o contato mais intenso com a pessoa com DM, tanto no atendimento nos grupos, quanto individualmente. Essa situação favorecia que a pessoa com DM pudesse esclarecer suas dúvidas e receber as orientações básicas, uma vez que o enfermeiro estava atento para essas necessidades.

Tem o acolhimento que geralmente é realizado por uma das enfermeiras [...] A gente precisa estar disponível também [...] o acompanhamento regular, quando ele está praticamente sem sintomas. Se o dia que ele não está bem eu não posso atendê-lo, daí o trabalho fica pela metade. (P07 MED C1) 


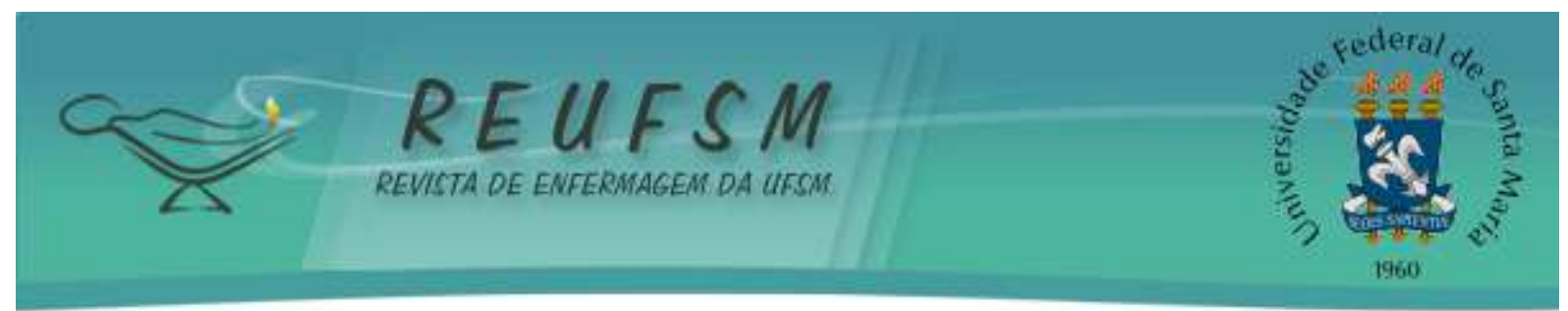

Havia dificuldade de efetuar o rastreamento para o diagnóstico precoce do DM e, também, daquelas pessoas que realizavam o acompanhamento fora do centro de saúde, principalmente pela falta de profissionais, em especial do Agente Comunitário de Saúde (ACS).

Então, a complicação da doença vai acontecer; mas, eu não falo tanto disso. Só quando a pessoa está bem descompensada, daí a gente conversa um pouco sobre a questão das amputações e tal [...] (P11 NUT C2)

Tenho certeza que tem muito caso com subdiagnóstico, paciente que tem diabetes, não sabe e a gente não tá descobrindo. Isso também porque a gente tem três microáreas descobertas, sem agente comunitário de saúde. (P12 ENF C3)

Os ACS trazem muita coisa para gente da comunidade. Bem importante isso. E ele, assim, em matéria de busca ativa, é praticamente tudo com ele. Essa ponte com a comunidade. Eles ajudam bastante; por isso, é importante as áreas cobertas. (P17 ENF C5)

A prevenção de complicações foi o assunto de maior impacto do estudo: não era uma realidade entre os profissionais, pois desenvolviam práticas pontuais e voltadas para situações específicas. Havia alguns relatos de preocupações com o "pé diabético" e as demais complicações não faziam parte de suas práticas. A visão que o profissional tinha do problema renal, por exemplo, era das pessoas com DM em situações em que a doença já estava instalada em estágio avançado, com o tratamento sendo realizado por um especialista ou no centro de referência onde realizava a hemodiálise.

Eu desconheço, aqui na minha área, pessoa em tratamento renal. Geralmente, esse paciente não vem muito na unidade de saúde. Ele faz o seu controle no centro de hemodiálise. É aquela coisa mais da atenção secundária. (P08 ENF C1)

Não, não tem nenhum em hemodiálise ou com doença renal crônica. Ainda não. Teoricamente, teriam que estar acompanhando com nefrologista. (P14 MED C4)

Os profissionais nem sempre tomavam como sua responsabilidade ações de prevenção das complicações, indicando que estas estavam na dependência das pessoas reconhecerem os sinais e sintomas e, então, buscarem o atendimento na atenção primária. Enfatizam que a prática de promoção da saúde precisa ser intersetorial, voltada à atividade física e à alimentação saudável, envolvendo a educação.

A promoção da saúde depende muito de um trabalho que seja intersetorial, com todos os profissionais envolvidos; precisa envolver a questão da educação [...] chega o assistente social aqui e trabalha 


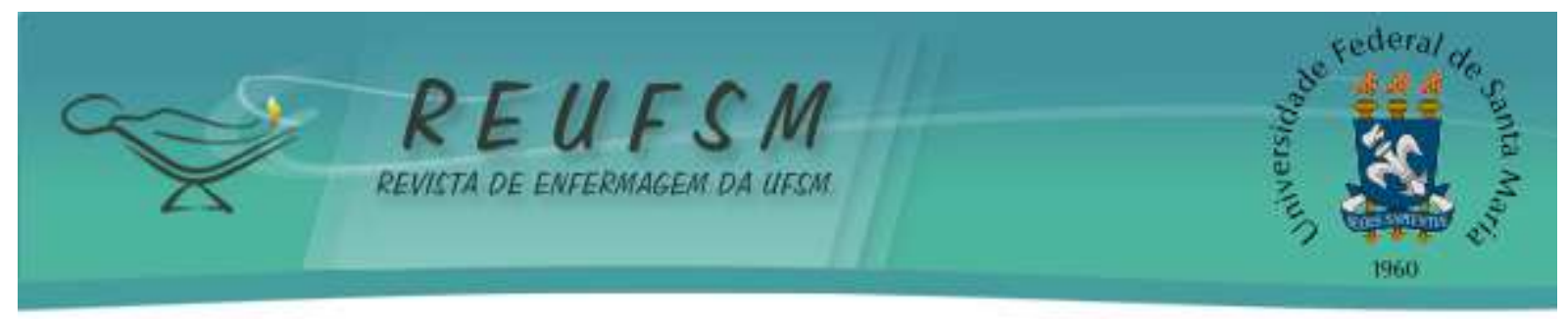

junto, entra em contato com o CRAS [Centros de Referência em Assistência Social] para aquela pessoa que não tem dificuldade venha até aqui e tal. Isso sim é trabalhar na promoção da saúde, mas ainda tem muito para avançar. (P11 NUT C2)

\section{DISCUSSÃO}

Mediante os resultados, ressalta-se a complexidade da integração entre saberes e práticas na atuação dos profissionais da saúde no cuidado a pessoa com DM e suas complicações.

Estudo que analisou o cuidado de pessoas com DM sob a ótica da integralidade por meio do acesso, do vínculo-responsabilização e da formação da equipe em um centro de saúde da Bahia, revelou que a atenção primária à saúde daquele município é pouco estruturada. Os resultados apontaram um modelo de organização dos serviços esgotado, um processo de cuidar construído no cotidiano do serviço de forma fragmentada, superficial, centrado no médico, provocando desestruturação e descontinuidade da atenção à saúde das pessoas com DM. ${ }^{18}$

No estado do Amazonas, estudo entrevistou profissionais de saúde, usuários do serviço e avaliou o Programa de Melhoria do Acesso e da Qualidade da Atenção Básica (PMAQ/AB) no ano de 2012, com o objetivo de descrever a organização do cuidado, especialmente direcionado a condições crônicas por equipes da ESF. ${ }^{19}$ Os resultados indicaram alta rotatividade de profissionais, baixa disponibilidade de insumos indispensáveis ao atendimento; organização fragmentada do cuidado; limitado uso de protocolos, planejamento e programação com base em critérios de risco e vulnerabilidade. ${ }^{19}$ Esses dados identificam-se com alguns aspectos do estudo, quando os profissionais reafirmam a fragilidade do cuidado integral e da integralidade do cuidado, voltado às queixas clínicas e sem busca ativa de pessoas com doenças crônicas devido à grande demanda.

Com o objetivo de compreender como os integrantes da ESF se auto organizam para a educação em saúde no manejo e na prevenção das complicações crônicas do DM, estudos identificaram problemas de relação entre os profissionais, dificuldades de planejamento e implantação das atividades educativas que incluem a orientação das pessoas para cuidar de sua saúde. Esses estudos enfatizam a necessidade dos profissionais de saúde buscarem tendências pedagógicas e não apenas realizar as atividades pautadas na sua experiência profissional. ${ }^{20-21}$ Achados como estes têm aproximações com o presente estudo, visto que, apesar do esforço profissional em realizar um atendimento interdisciplinar, não foi encontrado 


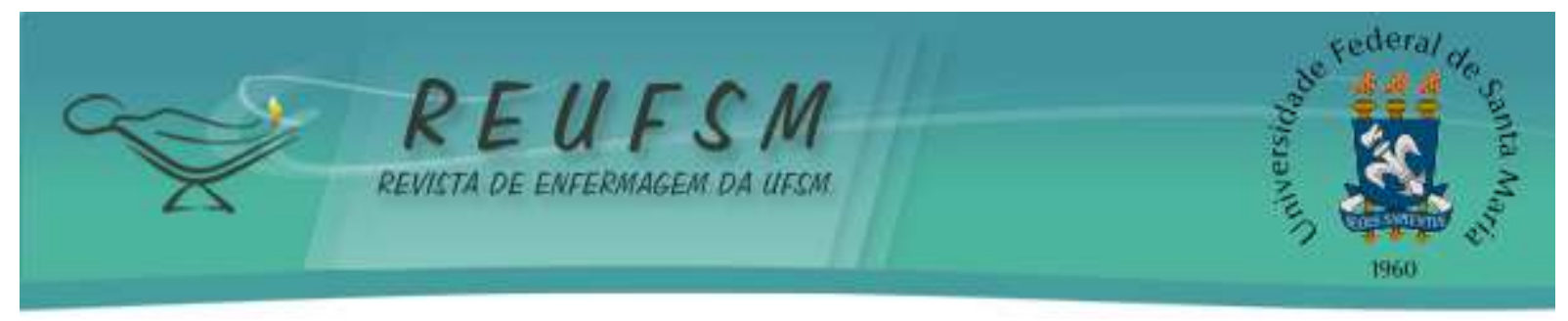

um serviço sistematizado e organizado onde a pessoa com DM transite entre as ações de educação em saúde e as redes de cuidado disponíveis no município.

Com intenção de analisar o conhecimento do usuário com DM sobre seus direitos de saúde, autores apontaram para o papel do poder público na divulgação de informações; as competências e responsabilidades dos profissionais de saúde; o dever ético-moral em informar; a participação ou a isenção dos usuários na busca por informações sobre seus direitos. E, mesmo com avanços nas políticas públicas, as pessoas com DM ainda desconhecem informações importantes sobre seus direitos. ${ }^{22}$

Os documentos do $\mathrm{MS}^{9-10}$ trouxeram importantes mudanças nas práticas de saúde, desde a criação e implantação do Sistema Único de Saúde, vigente no Brasil há mais de 20 anos. Nesse processo de implantação da uma nova política, houve a compreensão de que a mudança não aconteceria somente pela existência desses documentos, porque havia a necessidade de comprometimento e da formação dos profissionais da saúde. ${ }^{16}$

Neste estudo de um total de 22 profissionais entrevistados, 17 possuíam formação na área de Saúde da Família, o que foi atribuído ao fácil acesso a cursos de especializações, muitas vezes online, também ofertados por universidades federais e estaduais. Além desta formação, esperava-se contar com o interesse dos profissionais em aprimorarem seus conhecimentos técnicos, com a busca dos documentos que estão disponibilizados em sites do MS e/ou, ainda, impressos e distribuídos nos Centros de Saúde, além da gama de novas evidências científicas.

No entanto, conforme seus relatos, o excesso de atividades no dia a dia os leva a não estudarem tais documentos; alegam pouca disponibilidade para manterem-se atualizados. Outro destaque é que a estratégia de educação em saúde precisa ser melhor discutida e planejada para atender às necessidades específicas das pessoas com DM. ${ }^{23}$

O Caderno de Estratégias para o cuidado da pessoa com doença crônica ${ }^{9}$ e Estratégias para o cuidado da pessoa com doença crônica - Diabetes Mellitus, ${ }^{10}$ assim como o documento lançado em 2009, que estruturou a construção da Linha de Cuidado e Clínica Ampliada para Diabetes, ${ }^{20}$ foram as principais referências para os profissionais da saúde na atenção às pessoas com DM. Este último documento, ao orientar estratégias de atendimento que valorizam a integralidade, a equidade, a humanização, a resolubilidade, o controle social e a interdisciplinaridade, superando o modelo biomédico prevalente, parece ser a base para as ações dos profissionais entrevistados. 


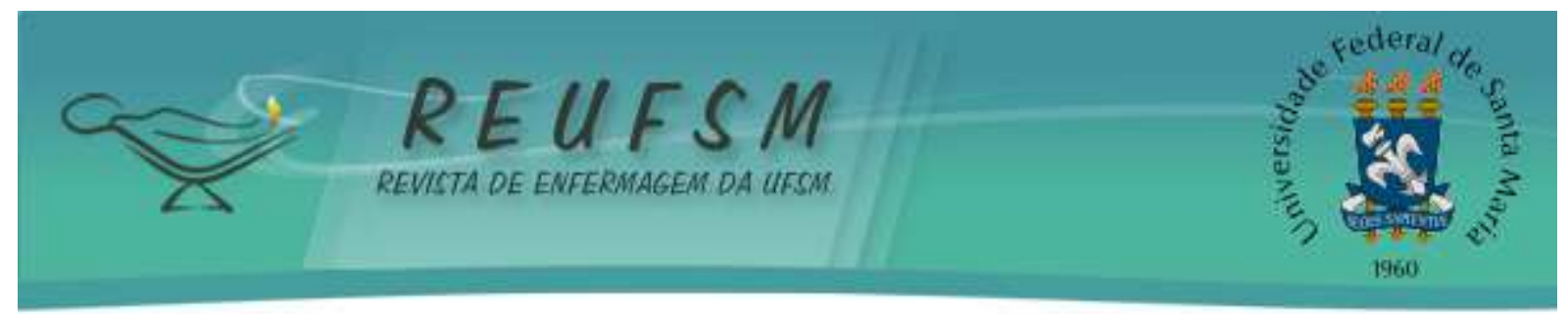

Houve, portanto, uma convergência entre o que os documentos indicam e as seguintes concepções: um atendimento voltado para a escuta; a criação e manutenção de vínculos e afetos; o reconhecimento de que as orientações que transmitem um sentimento de culpabilidade e transferência de responsabilidade não são boas aliadas na atenção ao usuário; o fato de descontruir o conceito que o conhecimento está centrado no profissional de saúde e valorizar o conhecimento da pessoa; a valorização da qualidade de vida; o uso de uma linguagem acessível/compreensível, que explique o que realmente a pessoa atendida possa compreender; por fim, a busca por um trabalho interdisciplinar entre a equipe de referência e a de apoio matricial.

\section{CONSIDERAÇÕES FINAIS}

Ao traçar um paralelo entre os saberes e as práticas dos profissionais de saúde na atenção à pessoa com DM e suas complicações, os resultados permitiram realçar que há muitos saberes convergentes com as políticas públicas. No entanto, a prática clínica aplicada à pessoa com DM exige reflexão e estruturação, a fim de retardar ou evitar as complicações dessa doença.

Os achados reforçaram o desafio constante na procura de estratégias avaliativas do processo de formação e implantação de políticas públicas em saúde e que, apesar da existência dessas, bem elaboradas e abrangentes, sua implantação ainda não é consolidada. Isso por motivos diversos; mas, especialmente, vinculados à falta de estrutura adequada nos serviços de atenção primária à saúde aliada a uma sobrecarga de atividades.

Quanto às práticas que vêm sendo desenvolvidas pelos profissionais nos centros de saúde para as pessoas com DM, este estudo evidenciou que o processo está em transição. Embora não ocorra uma atuação mais ampliada, que englobe a realização precoce do diagnóstico do DM, e também, a busca ativa/acompanhamento de pessoas com DM para acompanhamento sistemático, maior controle da doença e melhor prevenção das suas complicações, os profissionais demonstraram estar comprometidos com a qualidade dessa atenção e com a construção de novas ações, com vistas à promoção da saúde das pessoas atendidas na atenção primária.

Os saberes apresentaram maior proximidade com o que é preconizado por protocolos e guidelines do MS. Assim, os profissionais relataram o empenho em realizar um atendimento com abordagem diferenciada, englobando em um sistema de atenção e de cuidado integral e humanizado, com a preocupação em ter clareza sobre a real situação. Isso reforça a estratégia de abranger, voltando- 


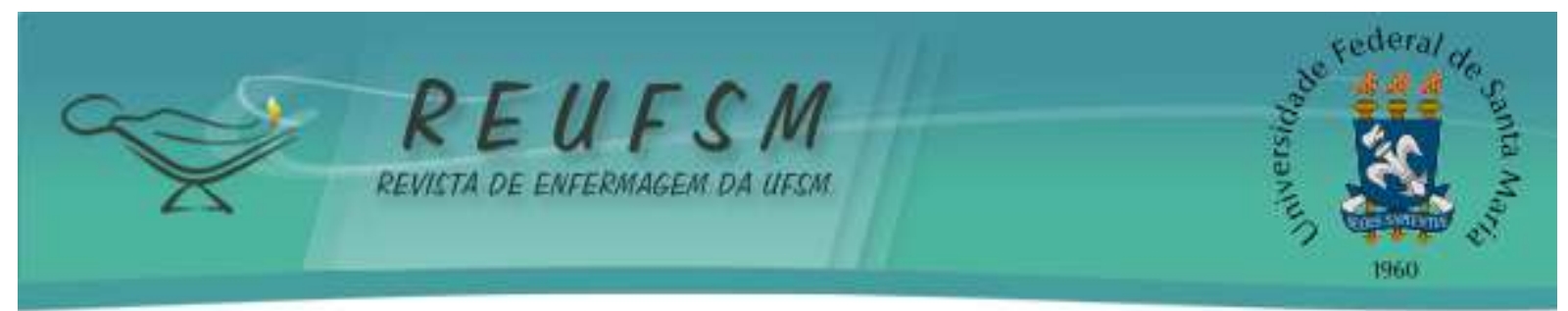

se para seu processo de vida. Nesta pesquisa, percebeu-se que os profissionais buscam a capacitação profissional e que isso transforma a maneira de eles olharem um indivíduo com DM.

Cabe ressaltar que todas essas práticas ainda não conseguem abordar tudo o que está proposto nas políticas de saúde. Dessa forma, são necessários avanços, em um esforço que não se restringe aos profissionais da saúde, porque envolve os gestores em todos os níveis de atuação e os usuários para o efetivo exercício da coparticipação e autonomia sobre seu processo saúde-doença.

O presente estudo contribui para a área da saúde e enfermagem, na medida em que, por meio da abordagem qualitativa, explora a complexidade do saber à prática no cuidado à pessoa com DM e suas complicações, e provoca uma reflexão sobre a prática e as políticas públicas instauradas. Logo, sugere-se que os órgãos governamentais e as instituições de saúde observem as ações para melhorar a estrutura dos serviços e a das equipes de saúde, apontadas como causas de divergências entre saberes e práticas.

Considera-se limitação do estudo o fato de ser realizado somente em uma cidade brasileira, conhecida referência nacional em qualidade de vida e na atenção primária à saúde.

\section{REFERÊNCIAS}

1. Brasil. Ministério da Saúde. Secretaria de Vigilância em Saúde. Departamento de Vigilância de Doenças e Agravos não Transmissíveis e Promoção da Saúde. Vigitel Brasil 2014: vigilância de fatores de risco e proteção para doenças crônicas por inquérito telefônico [Internet] Brasília (DF): Ministério da Saúde; 2015 [acesso em 2017 jun 1]. Disponível em: bvsms.saude.gov.br/bvs/publicacoes/vigitel_brasil_2014.pdf.

2. Garg SK, Hirsch IH. Self-monitoring of blood glucose - an overview. Diabetes Technology \&Therapeutics [Internet]. 2015 [acesso em 2016 ago 30];15(Supl 1):S-3-S-12. Disponível em: http://online.liebertpub.com/doi/pdf/10.1089/dia.2013.1501.

3. International Diabetes Federation (IDF), Siminerio L. Promoting diabetes care, prevention and a cure worldwide. 2014 [acesso em 2018 jun 30];21 slides: color. Disponivel em: https://www.joslin.org/Social_Responsibility_for_Diabetes_via_Community_Centric_Models _-_Linda_Siminerio_Robert_Gabbay.pdf.

4. American Diabetes Association (ADA). Standards of Medical Care in Diabetes - 2017. Diabetes Care [Internet]. 2017 [acesso em 2017 jan 10];40(Supl 1):1-135. Disponivel em: http://care.diabetesjournals.org/content/diacare/suppl/2016/12/15/40.Supplement_1.DC1/DC_ 40_S1_final.pdf.

5. Sociedade Brasileira de Diabetes (SBD); Grossi SAA, Pascali PM, organizadoras. Cuidados de enfermagem em Diabetes Mellitus. São Paulo; 2016. (Manual de enfermagem).

6. Oliveira AF, Marchi ACB de, Leguisano CP, Baldo GV, Wawginiak TA. Estimativa do custo de tratar o pé diabético, como prevenir e economizar recursos. Ciênc Saúde Colet. 


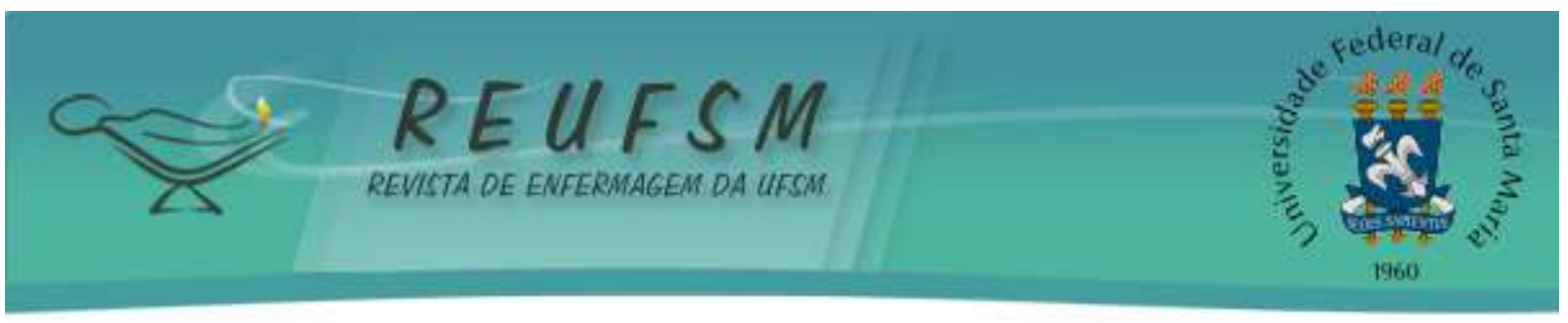

[Internet]. 2014 [acesso em 2016 ago 30];19(6):1663-71. Disponível em http://www.scielo.br/pdf/csc/v19n6/1413-8123-csc-19-06-01663.pdf.

7. Salci MA, Meirelles BHS, Silva DMGV. Primary care for Diabetes Mellitus patients from the perspective of the care model for chronic conditions. Rev Latinoam Enferm [Internet]. 2017 [acesso em 2017 jan 10];25:e2882. Disponível em: http://www.scielo.br/pdf/rlae/v25/pt_0104-1169-rlae-25-e2882.pdf.

8. Faria HTG, Santos MA dos, Arrelias CCA, Rodrigues FFL, Gonela JT, Teixeira CRS, et al. Adesão ao tratamento em Diabetes Mellitus em unidades da Estratégia Saúde da Família. Rev Esc Enferm USP [Internet]. 2014 [acesso em 2016 ago 30];48(2):257-63. Disponível em: http://www.scielo.br/pdf/reeusp/v48n2/pt_0080-6234-reeusp-48-02-257.pdf.

9. Brasil. Ministério da Saúde. Secretaria de Atenção à Saúde. Departamento de Atenção Básica. Estratégias para o cuidado da pessoa com doença crônica [Internet]. Brasília (DF): Ministério da Saúde; 2013 [acesso em 2016 ago 30]. Disponível em: http://bvsms.saude.gov.br/bvs/publicacoes/estrategias_cuidado_pessoa_doenca_cronica_cab3 5.pdf. (Cadernos de Atenção Básica; 35).

10. Brasil. Ministério da Saúde. Secretaria de Atenção à Saúde. Departamento de Atenção Básica. Estratégias para o cuidado da pessoa com doença crônica - Diabetes Mellitus [Internet]. Brasília (DF): Ministério da Saúde; 2013 [acesso em 2016 ago 30]. Disponível em http://189.28.128.100/dab/docs/portaldab/publicacoes/caderno_36.pdf. (Cadernos de Atenção Básica; 36).

11. Brasil. Ministério da Saúde. Portaria $n^{\circ}$ 4.279, de 30 de dezembro de 2010. Estabelece diretriz para a organização da Rede de Atenção à Saúde no âmbito do Sistema Único de Saúde [Internet]. Diário Oficial da União; 2010 [acesso em 2016 ago 30]. Disponível em: http://bvsms.saude.gov.br/bvs/saudelegis/gm/2010/prt4279_30_12_2010.html.

12. Brasil. Ministério da Saúde. Portaria $n^{\circ} 483$, de $1^{\circ}$ de abril de 2014. Redefine a Rede de Atenção à Saúde das Pessoas com Doenças Crônicas no âmbito do Sistema Único de Saúde (SUS) e estabelece diretrizes para a organização das suas linhas de cuidado [Internet]. Diário Oficial da União; 2014 abr 1 [acesso em 2016 ago 30]. Disponível em: http://bvsms.saude.gov.br/bvs/saudelegis/gm/2014/prt0483_01_04_2014.html.

13. Brasil. Ministério da Saúde. Secretaria de Atenção à Saúde. Departamento de Atenção Básica. Prevenção clínica de doenças cardiovasculares, cerebrovasculares e renais [Internet]. Brasília (DF): Ministério da Saúde; 2006 [acesso em 2016 ago 30]. Disponível em: http://bvsms.saude.gov.br/bvs/publicacoes/abcad14.pdf. (Cadernos de Atenção Básica;14).

14. Brasil. Ministério da Saúde. Secretaria de Atenção à Saúde. Departamento de Atenção Básica. Diabetes Mellitus [Internet]. Brasília (DF): Ministério da Saúde; 2006 [acesso em 2016 ago 30]. Disponível em: http://bvsms.saude.gov.br/bvs/publicacoes/diabetes_mellitus_cab16.pdf. (Cadernos de Atenção Básica;16).

15. Romanoski PJ. Saberes e práticas dos profissionais de saúde sobre a atenção às pessoas com diabetes mellitus [dissertação]. Florianópolis (SC): Universidade Federal de Santa Catarina; 2015. 


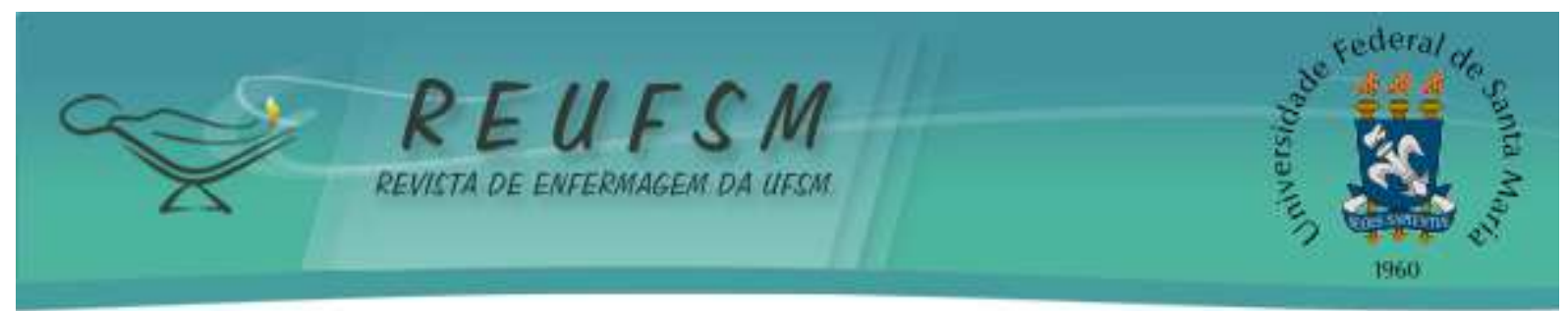

16. Hsieh HF, Shannon SE. Three approaches to qualitative content analysis. Qual Health Res [Internet]. 2005 [acesso em 2016 ago 30];15(9):1277-88. Disponível em: http://www.ncbi.nlm.nih.gov/pubmed/16204405.

17. Brasil. Ministério da Saúde. Conselho Nacional de Saúde. Resolução no 466, de 12 de dezembro de 2012. Aprova diretrizes e normas regulamentadoras de pesquisa envolvendo seres humanos [Internet]. Brasília: MS; 2012. [acesso em 2016 ago 30]. Disponível em: http://bvsms.saude.gov.br/bvs/saudelegis/cns/2013/res0466_12_12_2012.html.

18. Bastos LS, Assis MMA, Nascimento MAA, Oliveira LCF. Construção da integralidade no cuidar de pessoas com Diabetes Mellitus em um centro de saúde em Feira de Santana (BA). Ciênc Saúde Colet [Internet]. 2015 [acesso em 2015 set 15];16(Supl. 1):1417-26. Disponível em: http://www.scielo.br/pdf/csc/v16s1/a77v16s1.pdf.

19. Garnelo L, Lucas ACS, Parente RCP, Rocha ESC, Gonçalves MJF. Organização do cuidado às condições crônicas por equipes de Saúde da Família na Amazônia. Rio de Janeiro. Saúde Debate [Internet]. 2014 [acesso em 2016 out 31];38(N Esp):158-72. Disponível em: http://www.scielo.br/pdf/sdeb/v38nspe/0103-1104-sdeb-38-spe-0158.pdf.

20. Salci MA, Meirelles BHS, Silva DMGV. Primarycare for Diabetes Mellitus patientsfromthe perspective ofthecaremodel for chronicconditions. Rev Latinoam Enferm [Internet]. 2017 [acesso em 2016 nov 24];25:e2882. Disponivel em: http://www.scielo.br/pdf/rlae/v25/pt_0104-1169-rlae-25-e2882.pdf.

21. Roecker S, Nunes EFPA, Marcon SS. O trabalho educativo do enfermeiro na Estratégia Saúde da Família. Texto \& Contexto Enferm [Internet]. 2013 [acesso em 2018 fev 09];22(1):157-65. Disponível em: http://www.scielo.br/pdf/reeusp/v46n3/16.pdf.

22. Oliveira MSN, Almeida GBS, Chagas DDNP, Salazar PR, Ferreira LV. Autocuidado de idosos diagnosticados com hipertensão arterial e/ou diabetes mellitus. Rev Enferm UFSM [Internet]. 2017 [acesso em 2016 out 23];7(3):490-503. Disponível em https://periodicos.ufsm.br/reufsm/article/view/26344.

23. Campos TSP, Silva DMGV, Romanoski PJ, Ferreira C, Rocha FL. Fatores associados à adesão ao tratamento de pessoas com diabetes mellitus assistidos pela atenção primária de saúde. J Health Biol Sci [Internet]. 2016 [acesso em 2016 dez 22];4(4):251-6. Disponível em: http://periodicos.unichristus.edu.br/index.php/jhbs/article/view/1030/349.

Data de submissão: 23/01/2018

Data de aceite: 03/07/2018

E-mail: priscila.romanoski@gmail.com

Endereço: Rua Santino Raupp de Sá, 129 Apto 102 Costeira do Pirajubaé Florianópolis, Santa Catarina, Brasil.

CEP: 88047-220 